\title{
A method for unified optimization of systems and controllers
}

\section{Abildgaard, Ole}

Published in:

29th IEEE Conference on Decision and Control

Link to article, DOI:

10.1109/CDC.1990.204071

Publication date:

1990

Document Version

Publisher's PDF, also known as Version of record

Link back to DTU Orbit

Citation (APA):

Abildgaard, O. (1990). A method for unified optimization of systems and controllers. In 29th IEEE Conference on Decision and Control (Vol. Volume 4, pp. 2479-2480). IEEE. https://doi.org/10.1109/CDC.1990.204071

\section{General rights}

Copyright and moral rights for the publications made accessible in the public portal are retained by the authors and/or other copyright owners and it is a condition of accessing publications that users recognise and abide by the legal requirements associated with these rights.

- Users may download and print one copy of any publication from the public portal for the purpose of private study or research.

- You may not further distribute the material or use it for any profit-making activity or commercial gain

- You may freely distribute the URL identifying the publication in the public portal

If you believe that this document breaches copyright please contact us providing details, and we will remove access to the work immediately and investigate your claim 
Ole Abildgaard

\author{
Control Engineering Institute, Technical University of Denmark \\ Building 424, DK-2800 Lyngby, Denmark \\ Phone + 4545934419 EXT. 4523
}

\section{Abstract}

A unified method for solving control system optimization problems is suggested. All system matrices are allowed to be functions of the design variables. The method makes use of an implementation of a sequential quadratic programming algorithm (NLPQL) for solution of general constrained non-linear programming problems.

\section{Introduction}

The challenging problems, where the design variables are not limited to feedback gains and observer gains, but also may be plant parameters, are emphasized in this paper. Such unified optimization problems are important issues, especially in connection with active control of large space structures ([4] [7]). However, it turns out that the methods which are useful in the unified optimization problems also offer the possibility of optimization of less complex, but from a practical point of view very interesting cases, such as LQR output feedback (constant gain feedback from less than the full state vector), LQR design with eigenvalue equality and/or inequality constraints, and LQ-optimization of more classical controllers ([5]). The present formulation is computationally attractive, as it relies on the combined use of a robust numerical sequential quadratic programing algorithm and the widely used MATLAB package.

2. Unified optimization: Problem Formulation

Consider the n'th order LTI closed loop system:

$\dot{x}=\tilde{A} x \quad, \quad x(0)=x_{0} \quad, E\left\{x_{0} x_{0}^{T}\right\}=S$

and the objective function

$J=E\left\{\int_{0}^{\infty} x^{T} \tilde{Q} x d t\right\}$

where $\tilde{A}, \tilde{Q}$ and $S$ are matrix functions of the elements $\rho_{i}$ in the vector of design variables, ค. $E$ denotes expected value.

The following assumptions are made:

al: $\tilde{A}$ is a stability matrix

a2: $\tilde{Q}=\tilde{Q}^{T} \geq 0$

a3: $\{\tilde{A}, \tilde{D}\}$ is observable for any $\tilde{D}$ such that $\tilde{Q}=\tilde{D} \tilde{D}^{T}$

a : Eigenvalues of $\tilde{A}$ are distinct.

a5: The elements of $\tilde{A}$ and $\tilde{Q}$ are all continuously differentiable functions of the design variables in the domain of $\rho$.

Then with a1-a3 it is well known ([1]) that

$J=E\left\{x_{0}^{T} P x_{0}\right\}=\operatorname{tr}\left\{P E\left\{x_{0} x_{0}^{T}\right\}\right\}=\operatorname{tr}\{P S\}$

where $P$ is the unique (from al) solution of the matrix Lyapunov equation

$\lambda^{T} P+P \tilde{A}=-\tilde{Q}$

Let $\psi_{j}$ and $\phi_{j}$ be the bi-orthonormal left and

$\mathrm{CH} 2917-3 / 90 / 0000-2479 \$ 1.00$ ๑ 1990 IEEE right eigenvectors (normalized so that $\phi_{j}^{T} \phi_{j}=1$ ) corresponding to the j'th eigenvalue of $\tilde{A}, \lambda_{j}$. Then from a4 and as it can be shown ([4]) that $\frac{\partial \lambda_{j}}{\partial \rho_{i}}=\psi_{j}^{T}\left(\frac{\partial \tilde{A}}{\partial \rho_{i}}\right) \phi_{j}$

Furthermore, with al and as we can compute

$\frac{\partial J}{\partial \rho_{i}}=\operatorname{tr}\left\{\frac{\partial P}{\partial \rho_{i}} S+P \frac{\partial S}{\partial \rho_{i}}\right\}$

where $\frac{\partial P}{\partial p_{i}}$ is the unique (al) solution of the matrix Lyapunov equation ([5])

$\tilde{A}^{T} \frac{\partial P}{\partial \rho_{i}}+\frac{\partial P}{\partial \rho_{i}} \tilde{A}=-\left(\frac{\partial \tilde{Q}}{\partial \rho_{i}}+\frac{\partial \tilde{A}^{T}}{\partial \rho_{i}} P+P \frac{\partial \tilde{A}}{\partial \rho_{i}}\right)$

with (1)-(7) general nonlinear constrained optimization problems of the form

$\underset{p}{\text { Minimize }} F(\rho)$

$$
\begin{aligned}
& \text { Subject to } \quad G_{j}(\rho)=0, j=1, \ldots, M_{e} \\
& G_{j}(\rho) \geq 0, \quad j=M_{\mathrm{e}}+1, \ldots, M \\
& \rho_{i, L} \leq \rho_{i} \leq \rho_{i, u}, \quad i=1, \ldots, N
\end{aligned}
$$

can now be formulated and numerically solved. $F(\rho)$ may be any combination of objective functions like (2), (3) and eigenvalue assignment criteria ([5]). Likewise for $G_{i}(\rho)$ (the equality and inequality side constraints). The side constraints may be used to assure the assumptions al-a5 fulfilled during optimization. $\rho_{i, L}$ and $\rho_{i, U}$ are lower and upper bounds on the $i$ 'th design variable, respectively.

It should be noted, that the assumed stochastic nature of the initial condition distribution in (1) is essential in (3) in order to avoid specific initial condition dependence of the optimal solution. Another closely related approach leading to a worst case problem specification is presented in [3].

Since a specific control law is not assumed in (1), this method may be used very generally. special examples are the optimal tuning of classical P, PD, PI, PID controllers and of LQR output feedback controllers ([5]).

\section{NLPOL}

The program NLPQL is a FORTRAN implementation of a sequential quadratic programming method for solving general nonlinear programming problems, like (8). In each iteration step, a linearly constrained quadratic subproblem is formulated by approximating the Lagrange function quadratically and by linearizing the constraints. Subsequently, a one-dimensional line search is performed with respect to an augmented Lagrange merit function to obtain the new iterate. The merit function penalizes constraint violations. A further treatment of the algorithm and the flexibility it offers, can be found in [2].

In our implementation, MATLAB functions are 
used to compute the function values and gradients (from (3)-(7)) which are necessary inputs to NLPQL. The combination of NLPQL and MATIAB makes the necessary problem specific programming efforts relatively small ([5]).

It is worth noticing, that the equations (4) and (7) only differ on the right hand side, so it is possible to reuse the factorisations, which are used to solve (4), in the N solutions of (7).

\section{Example}

The system under consideration is a solid clamped - free Euler-Bernoulli beam of length $L=1$, density $d=1$ and Young modulus $E=1$ with a circular cross section. The design variables are the beam radius $r(x)$ (discretized into 40 elements of equal length), 2 positions of the collocated (point) force actuator/velocity sensor pairs and 4 feedback gains. Hence, the total number of design variables is 46 . The objective is to minimize the criterion

$J=E\left\{\int_{0}^{\infty}\left(x^{T} Q x+u^{T} R u\right) d t\right\}$

when the system is modelled as a n'th order LTI. The model is obtained through modal expansion and truncation $([6])$ :

$\dot{x}=A x+B u, \quad y=B^{T} x, \quad u=-G y$

$E\left\{x_{0} x_{0}^{T}\right\}=\frac{1}{n} Q^{-1} \Rightarrow E\left\{x_{0}^{T} Q x_{0}\right\}=1$

with

$$
\begin{aligned}
& A=\left[\begin{array}{cc}
0 & I \\
-\operatorname{diag}\left\{\omega_{1}^{2}\right\} & -\operatorname{diag}\left\{2 \zeta_{1} \omega_{1}\right\}
\end{array}\right], \quad B=\left[\begin{array}{c}
0 \\
B_{\frac{n}{2}}
\end{array}\right] . \\
& B_{\frac{n}{2}}=\left[\begin{array}{cc}
\phi_{1}\left(x_{1}\right) & \phi_{1}\left(x_{2}\right) \\
\cdot & \cdot \\
\cdot & \cdot \\
\phi_{\frac{n}{2}}\left(x_{1}\right) & \phi_{\frac{n}{2}}\left(x_{2}\right)
\end{array}\right] \quad G=\left[\begin{array}{ll}
g_{11} & g_{12} \\
g_{21} & g_{22}
\end{array}\right] \\
& Q=\left[\begin{array}{cc}
\operatorname{diag}\left\{\omega_{i}^{2}\right\} & 0 \\
0 & I
\end{array}\right] \quad R=0.01 \cdot I
\end{aligned}
$$

where $\zeta=0.04, \omega_{i}$ is the $i$ 'th modal eigenfrequency and $\phi_{i}$ is the corresponding normalized eigenfunction (modeshape). $n$ (eigenvalue) side constraints assure stability of the closed loop system and one side constraint limits the total volume to a maximum of 4 . The radius is limited to a maximum of 2 and a minimum of 0.2 . This yields (referring to (1) $-(3)$ ):

$\tilde{Q}=Q+B G^{T} R G B^{T} \quad \tilde{A}=A-B G B^{T} \quad S=Q^{-1}$

All matrices are continuously differentiable (no multiple eigenvalues) functions of $\rho$. The solutions obtained depend strongly on the initial choice of the two positions (cf. [8]), but a (local) optimum (for $n=6$ ) is found to be the beam designed as shown in fig. 1 with actuator/sensor positions

$x_{1}=0.77 \quad x_{2}=1.00$

and the gain matrix

$G=\left[\begin{array}{cc}5.03 & -0.33 \\ -1.22 & 2.71\end{array}\right]$

with $J=0.0702$ and volume $=4$.

This could be compared to the situation with a uniform beam of volume 4 with same actuator/sensor locations. Here the optimal gain matrix yields $J=0.1541$.

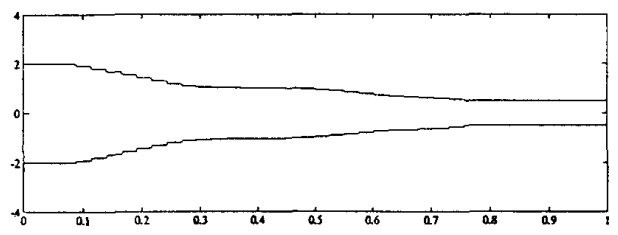

Fig. 1

Optimized beam

This result was obtained in 97 iterations in 3 hours at an Apollo 4000 workstation.

\section{Discussion}

Under weak conditions, the proposed method allows optimization of design variables in all system matrices. It uses a new approach, which is derived in this paper, with direct minimisation of the trace of the matrix product of the solution matrix from the Lyapunov equation for the system and the covariance matrix for the initial conditions. It has been shown how to compute the gradients of the objective function and the constraint functions imposing eigenvalue constraints. In an example it has been demonstrated how the method can solve a high dimensional problem, where the initial condition covariance assumption is used to assure constant initial mechanical energy in the beam during all iterations in the optimization. The initial energy is equally distributed in the modes, but this could easily be relaxed. Future work will examine the properties of the proposed method compared to other unified optimization approaches and the practical relevance of the objective function and the flexibility it offers.

\section{References}

[1] T. Kailath: "Linear Systems", PrenticeHall 1980

[2] K. Schittkowski: "NLPQL: A FORTRAN Subroutine Solving Constrained Nonlinear Programming Problems", Annals of operations Research, Vol. $51985 / 6$ pp. 485-500

[3] M.P. Bendsøe, N. Olhoff, J.E. Taylor: "On the Design of structure and Controls for optimal Performance of Actively Controlled Flexible Structures", Mech. of Str. \& Mach., vol. 15 1987, pp. 265-295

[4] K.B. Lim: "A Unified Approach to Structure and Controller Design optimizations", $\mathrm{Ph} . \mathrm{D}$ Dissertation, Virginia Polytechnic Institute and State University, Blacksburg, VA, 1986

[5] 0. Abildgaard: "Control system optimization: A General Approach Using an Imule= mentation of A sequential Quadratic Brogramming Algorithm", Control Engineering Institute, Technical university of Denmark, Rep. nr. S90.10, (1).1)

[6] L. Meirovitch: "Dynamics and Conlrul ir structures", Wiley-Interscience 1...)

[7] J.L. Junkins, D.W. Rew: "Unifisi ins i...: ation of Structures and controllers" in "Large Space Structures: Dyllamice alls control" (S.N. Atluri, A.K. Amu, 0.10, Springer Verlag 1988, pp. IA in

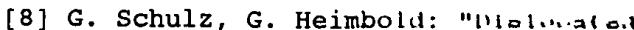
Actuator/Sensor Positiuntins ami r $00.11 . . .$.

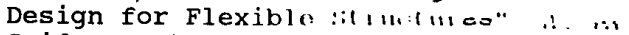
Guid., cont, and byu., $\times . .1=1=0, \mathrm{rr}$ 361-367 